Pacific

Journal of

Mathematics

COMPLEXITY AND HEEGAARD GENUS OF AN INFINITE CLASS OF COMPACT 3-MANIFOLDS Roberto Frigerio, Bruno Martelli, and Carlo Petronio 


\title{
COMPLEXITY AND HEEGAARD GENUS OF AN INFINITE CLASS OF COMPACT 3-MANIFOLDS
}

\author{
Roberto Frigerio, Bruno Martelli, and Carlo Petronio
}

\begin{abstract}
Using the theory of hyperbolic manifolds with totally geodesic boundary, we provide for every $n \geqslant 2$ a class $\mathcal{M}_{n}$ of such manifolds all having Matveev complexity equal to $n$ and Heegaard genus equal to $n+1$. All the elements of $\mathcal{M}_{n}$ have a single boundary component of genus $n$, and $\# \mathcal{M}_{n}$ grows at least exponentially with $n$.
\end{abstract}

This paper is devoted to the investigation of the class $\mathcal{M}_{n}$ of orientable compact 3-manifolds having an ideal triangulation with $n \geqslant 2$ tetrahedra and a single edge. We show in particular for each $M$ in $\mathcal{M}_{n}$ that the Heegaard genus of $M$ is equal to $n+1$, and that the complexity of $M$ in the sense of Matveev is equal to $n$. Moreover we prove that the classical invariants, such as homology and those of Turaev and Viro, cannot distinguish two different members of $\mathcal{M}_{n}$ from each other. However, using the fact that each $M$ in $\mathcal{M}_{n}$ carries a hyperbolic metric with totally geodesic boundary, we prove that $M$ has a unique ideal triangulation with $n$ tetrahedra. We exploit this property showing that the number of elements of $\mathcal{M}_{n}$ grows at least exponentially with $n$. This implies in particular the previously unknown fact that the rate of growth of the number of orientable boundary-irreducible acylindrical manifolds of complexity $n$ is also at least exponential in $n$. The class $\mathcal{M}_{n}$ was already considered in [8], but none of our results was covered there.

\section{Manifolds with a one-edged triangulation.}

In this section we introduce the class of manifolds we are interested in, and we prove their many remarkable topological and geometric properties.

Ideal triangulations and spines. We begin by recalling some definitions. An ideal tetrahedron is a tetrahedron with its vertices removed. An ideal triangulation of a compact 3-manifold $M$ with boundary is a realization of the interior of $M$ as a gluing of some ideal tetrahedra, induced by a simplicial pairing of the faces. A spine of $M$ is a compact polyhedron $P$ such that $M \backslash P=\partial M \times[0,1)$. A 2-dimensional polyhedron $Q$ is quasi-standard if every point has a neighbourhood homeomorphic to one of the polyhedra shown in Fig. 1. 


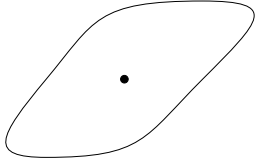

(1)

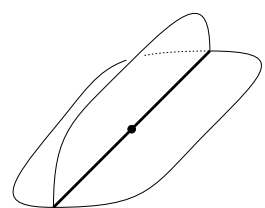

(2)

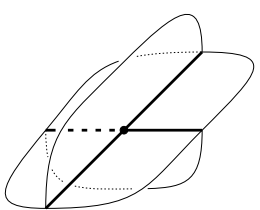

(3)

Figure 1. Local aspect of a quasi-standard polyhedron.

We denote by $V(Q)$ the set of points having regular neighbourhoods of type (3), and by $S(Q)$ the set of points having regular neighbourhoods of type (2) or (3). If $Q \backslash S(Q)$ consists of open cells and $S(Q) \backslash V(Q)$ consists of open edges, we say that $Q$ is standard, and we call faces the components of $Q \backslash S(Q)$. An ideal triangulation of $M$ defines in a natural way a dual standard polyhedron, which is in fact a spine of $M$ (see Fig. 2).
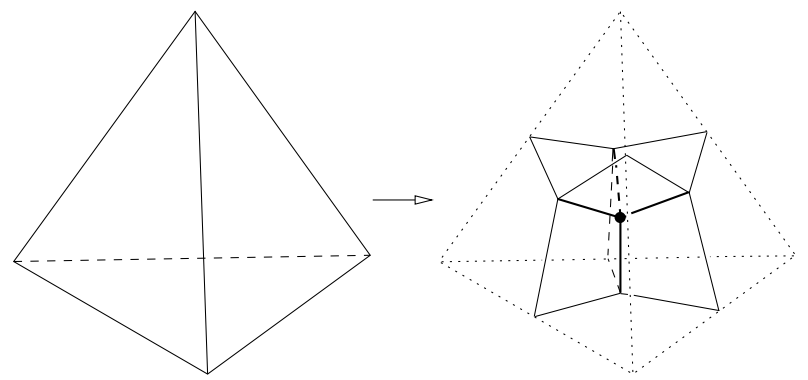

Figure 2. Duality between ideal triangulations and spines.

We now define the class of manifolds investigated in this paper. For every integer $n \geqslant 2$ we set

$\mathcal{M}_{n}=\{M: \operatorname{dim}(M)=3, M$ is compact and orientable, $\partial M \neq \emptyset$,

$M$ admits an ideal triangulation with one edge and $n$ tetrahedra\}.

The class $\mathcal{M}_{n}$ can be defined in various equivalent ways, as the next lemma shows.

Lemma 1.1. Let $M$ be a compact 3-manifold and let $\mathcal{T}$ be an ideal triangulation of $M$ consisting of $n$ tetrahedra. The following facts are equivalent:

1. $\mathcal{T}$ has one edge;

2. $\chi(M)=1-n$;

3. $\chi(M)=1-n$ and $\partial M$ is connected. 
Proof. Set $\widehat{M}=M / \partial M$, let $|\partial M|$ be the number of components of $\partial M$, and let $x$ be the number of edges of $\mathcal{T}$. We have $\chi(\widehat{M})=\chi(M)-\chi(\partial M)+|\partial M|$ and $\chi(\partial M)=2 \chi(M)$. Extending $\mathcal{T}$ to a cellularization of $\widehat{M}$, we also get

$$
\chi(\widehat{M})=|\partial M|-x+2 n-n=|\partial M|-x+n .
$$

Summing up, we get $\chi(M)=x-n$, which shows $(1) \Leftrightarrow(2)$.

We are left to show that if $x=1$ then $|\partial M|=1$. Since there is a single edge, we have $|\partial M| \leqslant 2$. Suppose $A$ and $B$ are distinct components of $\partial M$, and examine a triangular face $F$ of $\mathcal{T}$. Let $e_{1}, e_{2}$, and $e_{3}$ be the edges of $F$ viewed abstractly (i.e., before the embedding in $M$ ). Since $e_{1}, e_{2}, e_{3}$ become the same edge in $M$, they should all join $A$ to $B$, which is clearly impossible: If $e_{1}$ and $e_{2}$ join $A$ to $B$, then $e_{3}$ joins either $A$ or $B$ to itself.

Topological and geometric properties. Before proving our main theorem, we recall the definition of Matveev complexity of a compact 3-manifold with boundary, and the notion of hyperbolic 3-manifold with geodesic boundary. A compact 2-dimensional polyhedron $Q$ is said to be simple if the link of every point in $Q$ is contained in the 1-skeleton $K$ of the tetrahedron. (Note that a standard polyhedron is obviously simple.) A point having the whole of $K$ as a link is called a vertex, and its regular neighbourhood is as shown in Fig. 1-(3). This implies that the set $V(Q)$ of the vertices of $Q$ consists of isolated points, so it is finite. The complexity $c(M)$ of a compact 3 -manifold $M$ with boundary is the minimal number of vertices of a simple spine of $M$.

A hyperbolic 3-manifold with geodesic boundary is a complete Riemannian manifold with boundary which is locally isometric to a half-space of hyperbolic 3-space $\mathbb{H}^{3}$. By Mostow's Rigidity Theorem (see [6] for an explicit statement in the geodesic boundary case), every compact 3-manifold admits at most one hyperbolic structure with geodesic boundary, and has therefore a well-defined hyperbolic volume (if any). A useful tool for the computation of hyperbolic volumes, used in the sequel, is the Lobachevsky function $L: \mathbb{R} \rightarrow \mathbb{R}$ defined by

$$
L(\omega)=-\int_{0}^{\omega} \log |2 \sin u| \mathrm{d} u .
$$

To state our result we also recall that for any integer $r \geqslant 2$, after fixing $q_{0}$ in $\mathbb{C}$ such that $q_{0}^{2}$ is a primitive $r$-th root of unity, a real-valued invariant $\mathrm{TV}_{r}$ for compact 3-manifolds with boundary was defined by Turaev and Viro in [14]. We consider here these invariants normalized so that $\operatorname{TV}_{r}\left(S^{3}\right)=1$ 
for any $r$. Their computation involves the complex-valued quantum $6 j$ symbols $\left\{\begin{array}{ccc}i & j & k \\ l & m & n\end{array}\right\}$, where $i, j, k, l, m, n$ are half-integers, and the realvalued quantum integers $[k]=\frac{q_{0}^{k}-q_{0}^{-k}}{q_{0}-q_{0}^{-1}}$, defined for any integer $k$.

Theorem 1.2. Let $M \in \mathcal{M}_{n}$. Then:

1. $M$ is hyperbolic with geodesic boundary and its volume is given by

$$
\operatorname{vol}(M)=n \cdot\left(8 L\left(\frac{\pi}{4}\right)-3 \int_{0}^{\frac{\pi}{3 n}} \operatorname{arccosh}\left(\frac{\cos t}{2 \cos t-1}\right) \mathrm{d} t\right) .
$$

2. $M$ is boundary-irreducible and acylindrical.

3. Every closed embedded incompressible surface in $M$ is parallel to the boundary.

4. $H_{1}(M ; \mathbb{Z}) \cong \mathbb{Z}^{n}$.

5. The Heegaard genus of $M$ is equal to $n+1$.

6. $c(M)=n$.

7. The $r$-th Turaev-Viro invariant of $M$ is given by

$$
\operatorname{TV}_{r}(M)=\sum_{h \in \mathbb{N},}\left\{\begin{array}{lll}
h & h & h \\
h & h & h
\end{array}\right\}^{n} \cdot[2 h+1]^{1-n} .
$$

Before giving the Proof of Theorem 1.2, we introduce the notion of (hyperbolic) truncated tetrahedron $[\mathbf{7}, \mathbf{9}, \mathbf{6}]$. Let $\Delta$ be a tetrahedron and let $\Delta^{*}$ be the combinatorial polyhedron obtained by removing from $\Delta$ small open stars of the vertices. We call lateral hexagon and truncation triangle the intersection of $\Delta^{*}$ respectively with a face and with the link of a vertex of $\Delta$. The edges of the truncation triangles are called boundary edges, the other edges of $\Delta^{*}$ are called internal edges. A hyperbolic truncated tetrahedron is a realization of $\Delta^{*}$ as a compact polyhedron in $\mathbb{H}^{3}$, such that the truncation triangles are geodesic triangles, the lateral hexagons are geodesic hexagons, and truncation triangles and lateral hexagons lie at right angles to each other. A truncated tetrahedron is regular if all the dihedral angles along its internal edges are equal to each other. It turns out $[\mathbf{7}, \mathbf{6}]$ that for every $\theta$ with $0<\theta<\pi / 3$ there exists up to isometry exactly one regular truncated tetrahedron $\Delta_{\theta}^{*}$ of dihedral angle $\theta$. The boundary edges of $\Delta_{\theta}^{*}$ all have the same length $b=b(\theta)$, and the internal edges all have the same length $i=i(\theta)$, as shown in Fig. 3 .

The volume of $\Delta_{\theta}^{*}$ is given by (see $[\mathbf{1 3}]$ ):

$$
\operatorname{vol}\left(\Delta_{\theta}^{*}\right)=8 L\left(\frac{\pi}{4}\right)-3 \int_{0}^{\theta} \operatorname{arccosh}\left(\frac{\cos t}{2 \cos t-1}\right) \mathrm{d} t .
$$




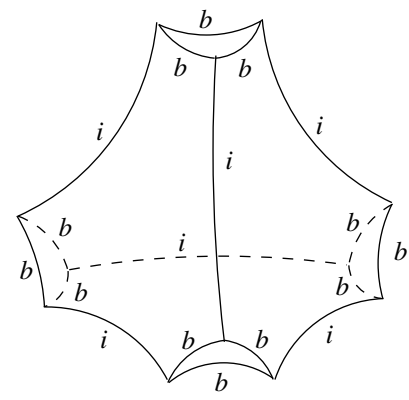

Figure 3. Length of the edges of a regular truncated tetrahedron.

As the Proof of Theorem 1.2 will now make clear, truncated tetrahedra can be used as building blocks to construct hyperbolic manifolds with geodesic boundary $[7,9,6]$.

Proof of Theorem 1.2. First of all, we fix a one-edged ideal triangulation $\mathcal{T}$ of $M$ and we denote by $e$ the single edge of $\mathcal{T}$.

In order to give $M$ a hyperbolic structure, we identify each tetrahedron of $\mathcal{T}$ with a copy of the regular truncated tetrahedron $\Delta_{\pi / 3 n}^{*}$. Due to the symmetries of $\Delta_{\pi / 3 n}^{*}$, every pairing between the faces of the tetrahedra of $\mathcal{T}$ can be realized by an isometry between the corresponding lateral hexagons of the $\Delta_{\pi / 3 n}^{*}$ 's. This procedure defines a hyperbolic metric on $M$, possibly with a cone singularity along $e$. But each tetrahedron is incident 6 times to $e$, so the cone angle around $e$ is $6 \cdot n \cdot \pi / 3 n=2 \pi$, and $M$ is actually hyperbolic without singularities. Finally, $\operatorname{vol}(M)=n \cdot \operatorname{vol}\left(\Delta_{\pi / 3 n}^{*}\right)$ and Point (1) is proved.

Point (2) is a general consequence of the existence of a hyperbolic structure with geodesic boundary, and Point (3) is proved using Haken's theory of normal surfaces. If $\Sigma \subset M$ is a closed incompressible surface, then $\Sigma$ can be isotoped into normal position with respect to $\mathcal{T}$, so the intersection of $\Sigma$ with an ideal tetrahedron $T \in \mathcal{T}$ consists of triangles and squares. Recall now that $\Sigma$ intersects all the edges of $T$ in the same number $k$ of points. Assuming there are $q$ squares and $t_{i}$ triangles of type $i$, for $i=1, \ldots, 4$, we deduce the relations $t_{1}+t_{2}=t_{3}+t_{4}=t_{1}+t_{3}+q=t_{1}+t_{4}+q=t_{2}+t_{3}+q=t_{2}+t_{4}+q=k$. This easily implies that $q=0$ and $t_{i}=k / 2$ (so $k$ is even), hence $\Sigma \cap T$ consists of $k / 2$ parallel triangles at each vertex of $T$. Therefore $\Sigma$ consists of $k / 2$ disjoint surfaces parallel to $\partial M$.

Concerning Point (4), let $P$ be the standard spine of $M$ dual to $\mathcal{T}$. Since $M$ collapses onto $P$, we have $H_{1}(M ; \mathbb{Z}) \cong H_{1}(P ; \mathbb{Z})$, and we can use cellular homology to compute $H_{1}(P ; \mathbb{Z})$. To do so, we choose a maximal tree $Y$ in the 4 -valent graph $S(P)$. Then $S(P) \backslash Y$ consists of $n+1$ edges $e_{1}, \ldots, e_{n+1}$. 
Choose an orientation on each $e_{i}$ and on the single face $F$ of $P$. The face $F$ is incident three times to each $e_{i}$. For $i=1, \ldots, n+1$ let $r_{i}$ be the sum of a contribution \pm 1 over the three instances $F$ runs along $e_{i}$, with sign depending on whether orientations are matched or not. So $r_{i} \in\{-3,-1,1,3\}$ and $H_{1}(P ; \mathbb{Z}) \cong \mathbb{Z}^{n+1} /\langle r\rangle$ where $r=\left(r_{1}, \ldots, r_{n+1}\right)$. We will now prove that $r_{i}= \pm 1$ for some $i$, which implies that $H_{1}(P ; \mathbb{Z}) \cong \mathbb{Z}^{n}$. Let $v \in V(P)$ be an extremal vertex of $Y$, i.e., a vertex adjacent to only one edge of $Y$, whence to three of the $e_{i}$ 's, say $e_{i_{1}}, e_{i_{2}}, e_{i_{3}}$ (indices could be repeated according to multiplicity of incidence). Looking at the picture of the neighbourhood of $v$ in $P$, shown in Fig. 1-(3), one now easily sees that $F$ cannot be incident three times in the same direction to each $e_{i_{1}}, e_{i_{2}}, e_{i_{3}}$, so we have $r_{i}= \pm 1$ for some $i \in\left\{i_{1}, i_{2}, i_{3}\right\}$.

Let us turn to Point (5). Lemma 1.1 shows that $\partial M$ has genus $n$ but, by Point (2), $M$ is not a handlebody, so its genus is at least $n+1$. A Heegaard surface having genus $n+1$ is simply given by the boundary of a regular neighbourhood of $\partial M \cup e$, whence the conclusion.

We now prove Point (6). The standard spine $P$ dual to $\mathcal{T}$ has $n$ vertices, so we have $c(M) \leqslant n$. By Point (2), a result of Matveev [11] shows that there is a standard spine $Q$ of $M$ (not just a simple one) with precisely $c(M)$ vertices. An Euler characteristic computation gives $\chi(Q)=x-c(M)$ where $x \geqslant 1$ is the number of faces of $Q$, so $c(M) \geqslant 1-\chi(Q)=1-\chi(M)=n$, the last equality having been proved in Lemma 1.1.

Point (7) is an easy calculation. We follow the notation of [14]: Since there is only one face $F$, a colouring of $P$ is given by assigning to $F$ a half-integer in $\{0,1 / 2,1,3 / 2, \ldots,(r-2) / 2\}$. Since the same $F$ is incident three times to each edge, the colouring is admissible if it is an integer $h$ with $0 \leqslant 3 h \leqslant r-2$. Each such colouring contributes to $\mathrm{TV}_{r}(M)$ with a summand given by the product of a factor $\left\{\begin{array}{lll}h & h & h \\ h & h & h\end{array}\right\} \cdot[2 h+1]^{-1}$ for each vertex and of a factor $[2 h+1]$ due to the single face of $P$.

Remark 1.3. The Proof of Theorem 1.2-(1) actually shows that every oneedged ideal triangulation of $M \in \mathcal{M}_{n}$ is combinatorially equivalent to a decomposition of $M$ into $n$ regular truncated tetrahedra of dihedral angle $\pi / 3 n$.

Remark 1.4. It was proved in [13] that among compact hyperbolic 3 manifolds with geodesic boundary and fixed Euler characteristic $\chi<0$, those having minimal volume are decomposed into $1-\chi$ copies of $\Delta_{\pi / 3(1-\chi)}^{*}$. Therefore $\mathcal{M}_{n}$ is precisely the set of hyperbolic 3 -manifolds $M$ with geodesic boundary having minimal volume among compact orientable manifolds with $\chi(M)=1-n$. 
Remark 1.5. It follows from the Proof of Theorem 1.2-(6) that $\mathcal{M}_{n}$ is also the set of all hyperbolic manifolds $M$ having minimal complexity among compact orientable manifolds with $\chi(M)=1-n$.

Remark 1.6. Point (7) of Theorem 1.2 shows in particular that $\mathrm{TV}_{r}(M)$, for $M$ in $\mathcal{M}_{n}$, depends only on $n$ and $r$ (actually, on $q_{0}$ ), but not on $M$. This could also be proved using the fact that two standard spines with the same incidence relations between faces and vertices produce the same Turaev-Viro invariants (see [12] for more details). In fact, each manifold in $\mathcal{M}_{n}$ admits a spine with one face and $n$ vertices, so the incidence relations are always the same.

Remark 1.7. We believe that Theorem 1.2 extends, with minor variations, to non-orientable manifolds admitting a one-edged triangulation. However, to prove this extension, one should first generalize Matveev's theorem [11] on standardness of minimal spines to the case of non-orientable, boundaryirreducible manifolds not containing projective planes or essential Möbius strips.

Uniqueness of the minimal spine. We are now left with two tasks: We must provide examples of manifolds in $\mathcal{M}_{n}$, and we must be able to distinguish manifolds in the same $\mathcal{M}_{n}$. We will face the former task in the next section, by constructing standard polyhedra with $n$ vertices and one face. Concerning the latter task, we have just shown that homology, Heegaard genus, Turaev-Viro invariants, and volume of manifolds in $\mathcal{M}_{n}$ depend on $n$ only, so we need a more powerful tool. This tool is provided by hyperbolic geometry. We recall that the cut-locus of a hyperbolic manifold $M$ with geodesic boundary is the set of all points of $M$ which admit at least two distinct distance-minimizing geodesics to $\partial M$.

Theorem 1.8. Every $M \in \mathcal{M}_{n}$ has a unique standard spine with $n$ vertices, homeomorphic to the cut-locus of $M$.

Proof. Let $C \subset M$ be the cut-locus of $M$, and let $P$ be a standard spine of $M$ with $n$ vertices. By Remark 1.3, $P$ is dual to a decomposition $\mathcal{T}$ of $M$ into $n$ regular truncated tetrahedra. We claim that $C$ intersects each tetrahedron $T \in \mathcal{T}$ as in Fig. 2-right. This implies that $C$ is homeomorphic to $P$, whence the conclusion.

To prove our claim it is sufficient to show that for every tetrahedron $T$ of $\mathcal{T}$, every point $p$ in $T$, and every distance-minimizing geodesic $\gamma$ connecting $p$ to $\partial M$, we have that $\gamma$ is entirely contained in $T$. If this were not true, since $\gamma$ meets $\partial M$ at a right angle, a subarc $\gamma^{\prime}$ of $\gamma$ would connect a truncation triangle and its opposite hexagon in some tetrahedron $T^{\prime} \in \mathcal{T}$. Then the length of $\gamma^{\prime}$ would be greater than the distance between such a truncation triangle and its opposite hexagon, which in turn is greater than the distance 
between $p$ and some truncation triangle in $T$, since $T$ and $T^{\prime}$ are isometric to each other and regular.

Remark 1.9. An alternative proof of Theorem 1.8 could be based on the machinery developed in [6]: Remark 1.3 and the tilt-formula $[\mathbf{1 6}, \mathbf{1 5}, \mathbf{6}]$ easily imply that a one-edged ideal triangulation of a manifold $M \in \mathcal{M}_{n}$ is combinatorially equivalent to Kojima's canonical decomposition of $M$, which is obtained by straightening the ideal triangulation dual to the cut-locus of $M$ (see [9]).

We say that a standard polyhedron is orientable if it can be embedded in an orientable 3-manifold. Note that an orientable standard polyhedron is automatically the spine of an orientable 3 -manifold, which is unique by [3].

Corollary 1.10. The set $\mathcal{M}_{n}$ is in one-to-one correspondence with the set of orientable standard polyhedra with $n$ vertices and one face.

Automorphisms and chirality. Another remarkable consequence of Theorem 1.8 is that there is an easy algorithm running in $n^{2}$ time to determine all the automorphisms of an element $M$ of $\mathcal{M}_{n}$. This algorithm will check in particular whether $M$ is amphichiral or chiral, i.e., whether $M$ admits an orientation-reversing automorphism or not. The algorithm is based on the following consequence of Theorem 1.8:

Corollary 1.11. The automorphisms of $M \in \mathcal{M}_{n}$ correspond bijectively to the combinatorial automorphisms of the triangulation dual to the unique minimal spine of $M$.

The algorithm now works as follows: First, pick an arbitrary "base" tetrahedron $T_{0}$ in the ideal triangulation $\mathcal{T}$ of $M$. Second, for each $T$ in $\mathcal{T}$, consider the 24 combinatorial isomorphisms $f: T_{0} \rightarrow T$ (12 of which are orientation-reversing). Third, check whether $f$ extends to the whole of $\mathcal{T}$. The process of checking whether a given $f$ extends is linear in $n$, so the whole algorithm runs in time proportional to $n^{2}$.

\section{Numerical estimates.}

The results of the previous section would of course be of little or no interest if $\mathcal{M}_{n}$ (the class of manifolds having a triangulation with one edge and $n$ tetrahedra) turned out to be empty or very small. In this section we prove that $\# \mathcal{M}_{n}$ grows at least exponentially with $n$, deducing that the number of orientable compact 3-manifolds of complexity $n$ also grows at least exponentially (Corollary 2.6). We do this by concentrating on a special class of one-edged triangulations, and we give some hints showing that our exponential lower estimate on $\# \mathcal{M}_{n}$ is actually far from being sharp. ${ }^{1}$

\footnotetext{
${ }^{1}$ Added in proof. We have actually shown in math.GT/0301114 that $\# \mathcal{M}_{n}$ has growth type $n^{n}$.
} 
Oriented spines and o-graphs. In the whole of this section we consider oriented (rather than just orientable) manifolds (but recall that chirality of the elements of $\mathcal{M}_{n}$ can be checked very easily). We remind [1] that if $P$ is a standard spine of an oriented $M$ then $P$ also carries an orientation, defined as a screw-orientation along the edges of $S(P)$ with a natural compatibility at vertices (see [2, Fig. 2]). Conversely, if $P$ is an oriented standard polyhedron, then $P$ is orientable, and the manifold it defines is oriented. In addition, $P$ can be described by two additional structures on the 4-valent graph $S(P)$ :

- An embedding in the plane of the neighbourhood of each vertex, with two opposite strands marked as being over the other two, as in knot projections.

- A colour in $\mathbb{Z} / 3$ attached to each edge.

A 4-valent graph with these additional structures is called an o-graph. It was shown in [1] that any o-graph defines an oriented standard polyhedron, whence an oriented manifold, and that two o-graphs defining the same oriented polyhedron are related by certain "C-moves." The effect of a C-move is to change the planar structure at a vertex and the $\mathbb{Z} / 3^{\text {-colouring of the }}$ edges incident to this vertex.

O-graphs based on the open chain. Let $G_{n}$ be the graph with vertices $v_{1}, \ldots, v_{n}$, a closed edge at $v_{1}$ and one at $v_{n}$, and two edges joining $v_{i}$ to $v_{i+1}$ for $i=1, \ldots, n-1$. We characterize in this paragraph the oriented standard polyhedra $P$ such that $S(P)=G_{n}$ and $P$ has a single face. We begin with the following fact that one can readily establish using the $\mathrm{C}$-moves mentioned above:

Lemma 2.1. Any oriented standard polyhedron $P$ such that $S(P)=G_{n}$ can be represented by an o-graph as shown in Fig. 4.

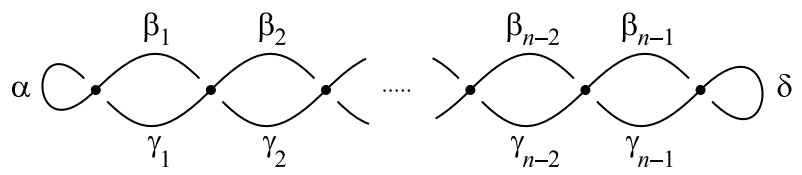

Figure 4. O-graph of a generic polyhedron based on $G_{n}$.

The description of which o-graphs as in Fig. 4 have a single face will use the language of finite state automata (see for instance [4]). We recall that a finite state automaton over a finite set $A$ (the alphabet) consists of a finite set $S$ (the states), a function $S \times A \rightarrow S$ (the transition function), an element $s_{0}$ of $S$ (the start state), and a subset $S^{\prime}$ of $S$ (the set of accept states). A word (a finite string of letters from the alphabet) is accepted 
by the automaton if, starting from $s_{0}$, reading the word from left to right, and using the transition function, the automaton ends in a state of $S^{\prime}$. An automaton can be encoded by a picture, where the states are represented by $S$-labeled boxes (with double margin for accept states), the transition function is given by box-to-box $A$-labeled arrows, and a mark indicates the start state.

Theorem 2.2. The oriented standard polyhedron defined by the o-graph of Fig. 4 has a single face if and only if both $\alpha$ and $\delta$ are different from 2 and the word

$$
\left(\beta_{1}, \gamma_{1}\right)\left(\beta_{2}, \gamma_{2}\right) \ldots\left(\beta_{n-1}, \gamma_{n-1}\right)
$$

is accepted by the automaton described in Fig. 5, where

$$
\begin{aligned}
& A_{0}=\{(2,2)\}, \quad A_{1}=\{(0,0),(1,1)\}, \quad A_{2}=\{(1,0),(0,1)\}, \\
& A_{3}=\{(0,2),(1,2),(2,0),(2,1)\}, \quad A=A_{0} \cup A_{1} \cup A_{2} \cup A_{3} .
\end{aligned}
$$

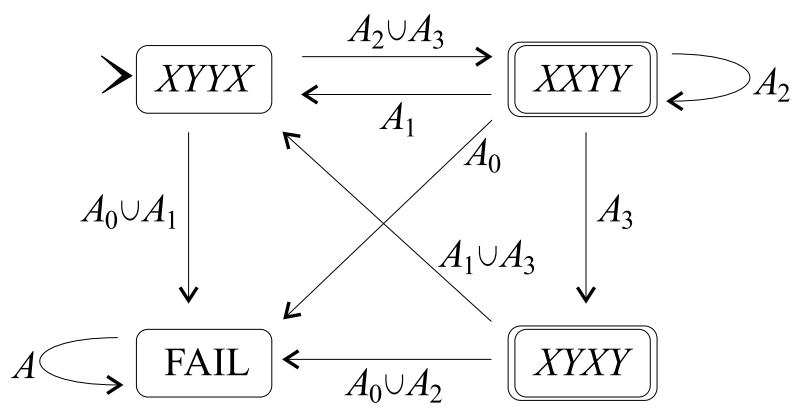

Figure 5. A finite state automaton with alphabet $A=A_{0} \cup$ $A_{1} \cup A_{2} \cup A_{3}$.

Proof. We confine ourselves to a general explanation, omitting the many combinatorial details. We analyze the graph left to right, starting from $\alpha$.
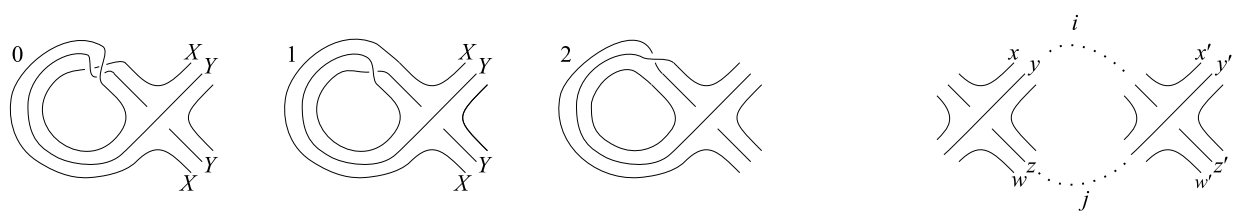

Figure 6. Portions of o-graph.

Figure 6 shows that for $\alpha=2$ there are at least two faces, so $\alpha \in\{0,1\}$. Now we examine the pair of colours $\left(\beta_{1}, \gamma_{1}\right)$ starting with the string $X Y Y X$ 
which describes the way the faces already constructed are matched to the left of the point we are considering. (Note that both $\alpha=0$ and $\alpha=1$ give $X Y Y X$.) Depending on $\left(\beta_{1}, \gamma_{1}\right)$ we will have either the creation of a face which does not fill the polyhedron, or a new pattern that describes the matching of faces. More generally, as we proceed, we will have a string $x y z w$ of two symbols each repeated twice, and we will have to analyze the effect of a pair of colours $(i, j)$, which either creates a closed face or produces a new pattern $x^{\prime} y^{\prime} z^{\prime} w^{\prime}$ (see Fig. 6 again). The detailed analysis of all the possibilities leads precisely to the transitions shown in Fig. 5, where "FAIL" means that a closed face is created. To conclude we note that the final edge with colour $\delta \in\{0,1\}$ gives a single global face when the input pattern is $X X Y Y$ or $X Y X Y$, and not otherwise.

Growth of $\# \mathcal{M}_{n}$. Theorem 1.8 shows that to compute $\# \mathcal{M}_{n}$ it is sufficient to count the combinatorially distinct standard polyhedra with $n$ vertices and one face. Restricting to the oriented ones with the open chain $G_{n}$ as singular graph we must then discuss which o-graphs as in Fig. 4 define the same polyhedron. A move that of course does not change the polyhedron associated to the o-graph is the $180^{\circ}$ degree rotation. Using the $\mathrm{C}$-moves of $[\mathbf{1}]$ one can see that another such move consists in interchanging each $\beta_{k}$ with the corresponding $\gamma_{k}$. In addition, these two moves are sufficient to generate all graphs giving the same polyhedron. Therefore we have the following:

Lemma 2.3. An oriented standard polyhedron is defined by at most four different o-graphs as in Fig. 4.

Proposition 2.4. There are at least $4 \cdot 12^{(2 n-5) / 3}$ distinct oriented standard polyhedra with one face and the open chain with $n$ vertices as singular set.

Proof. We must count the possible choices for the $\beta_{k}$ 's and $\gamma_{k}$ 's, i.e., the words of length $n-1$ accepted by the finite state automaton of Fig. 5, multiply by 4 (the choices for $\alpha$ and $\delta$ ), and divide by at most 4 according to the previous lemma. So it is sufficient to prove that there are at least $4 \cdot 12^{(2 n-5) / 3}$ words of length $n-1$ accepted by the automaton.

The idea is just to perform the loop $X Y Y X \rightarrow X X Y Y \rightarrow X Y X Y \rightarrow$ $X Y Y X$ in all possible ways, inserting a single loop $X X Y Y \rightarrow X X Y Y$ when $n-1$ is a multiple of 3 (for in this case we would end up in the nonaccept start state). Since 6 letters lead from $X Y Y X$ to $X X Y Y, 4$ lead from $X X Y Y$ to $X Y X Y$, and again 6 from $X Y X Y$ to $X Y Y X$, it is clear that approximately $6^{2(n-1) / 3} \cdot 4^{(n-1) / 3}=12^{2(n-1) / 3}$ words can be constructed with this method. The exact computation carried out depending on the congruence class of $n-1$ modulo 3 leads to the desired estimate. 
The next easy remark shows that the qualitative type of growth just established is actually the maximal one could expect. After the remark we also give an obvious consequence of the previous proposition.

Remark 2.5. Given a 4 -valent graph $G$ with $n$ vertices, there exist at most $2^{n} \cdot 3^{2 n}=18^{n}$ distinct oriented standard polyhedra $P$ such that $S(P)=G$. If $G=G_{n}$, using Lemma 2.1, one can see that there exist at most $3^{2 n}=9^{n}$ of them.

Corollary 2.6. There exist $c>0$ and $b>1$ such that $\# \mathcal{M}_{n} \geqslant c \cdot b^{n}$. In particular, the number of distinct orientable, boundary-irreducible, and acylindrical manifolds of complexity $n$ is at least $c \cdot b^{n}$.

We remind the reader that the manifolds referred to in the previous corollary are precisely those known to have standard minimal spines. Now we have:

Remark 2.7. The number of distinct oriented standard polyhedra with $n$ vertices is bounded from above by $18^{n} \cdot g(n)$, where $g(n) \leqslant(4 n-1)$ !! is the number of distinct four-valent graphs with $n$ vertices.

Further comments on estimates. The lower bound on the number of elements of $\mathcal{M}_{n}$ based on the open chain graph $G_{n}$ provided by Proposition 2.4 is very far from being sharp. For instance, if we consider in Fig. 5 the paths consisting of some $X Y Y X \rightarrow X X Y Y \rightarrow X Y X Y \rightarrow X Y Y X$ cycles intermingled with some $X X Y Y \rightarrow X X Y Y$ loops, we deduce that the number of distinct spines is at least

$$
\sum_{k=0}^{n-2} \begin{cases}0 & \text { if } n-k-1=3 h, \\
2^{k} \cdot 6^{2 h+1} \cdot 4^{h} \cdot\left(\begin{array}{c}
h+k \\
h
\end{array}\right) & \text { if } n-k-1=3 h+1, \\
2^{k} \cdot 6^{2 h+1} \cdot 4^{h+1} \cdot\left(\begin{array}{c}
h+k \\
h
\end{array}\right) & \text { if } n-k-1=3 h+2 .\end{cases}
$$

Concentrating on the term of the sum corresponding to $k=[n / 7]$ and using Stirling's formula one can for instance deduce from this estimate that there exists $c>0$ such that, for all $\varepsilon>0$, the number of oriented elements of $\mathcal{M}_{n}$ based on $G_{n}$ is at least $c \cdot(6-\varepsilon)^{n}$ for $n \gg 0$. Note that $12^{2 / 3} \cong 5.2418$.

Remark 2.8. With the aid of a computer, in $[\mathbf{1 0}]$ we have listed and classified the approximately 2,000 closed, irreducible and orientable manifolds of complexity up to 9 . Corollary 2.6 suggest that a similar listing for orientable, compact, boundary-irreducible, acylindrical manifolds may be hopeless. Our lower bound on their number, even if not sharp, already implies that there are at least 115,000 such manifolds of complexity up to 9 . 
Another example: The closed chain. We have concentrated in this section on the open chain $G_{n}$, because this graph is already sufficient to show that $\# \mathcal{M}_{n}$ grows exponentially. But we believe that a systematic investigation of the 4-valent graphs supporting a polyhedron with a single face would be quite interesting. Our guess is actually that most graphs indeed support many different such polyhedra. As another example we only mention the closed chain, shown in Fig. 7.

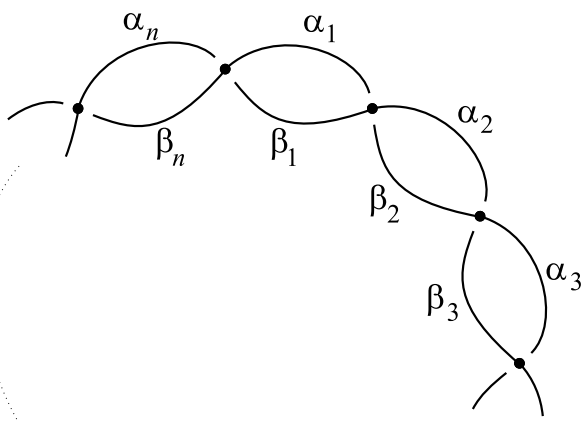

Figure 7. An o-graph based on the closed chain.

The combinatorial analysis is in this case harder than that carried out for Theorem 2.2, but we state at least the following fact, which already implies that again in this case the number of relevant polyhedra grows exponentially with $n$ :

Proposition 2.9. Fix $\left(\alpha_{1}, \beta_{1}\right) \in\{(0,0),(0,1),(1,0),(1,1)\}$ in the o-graph of Fig. 7. Let $\left(\alpha_{2}, \beta_{2}\right) \ldots\left(\alpha_{n}, \beta_{n}\right)$ be a random word $w$ in the letters $\{(0,2)$, $(1,2),(2,0),(2,1)\}$. Then for $n \gg 0$ there is a probability $1 / 2$ that $w$ defines a polyhedron with a single face.

Chirality. We get back here to the chirality issue discussed at the end of Section 1, showing how o-graphs can be used in connection with it. We first recall [1] that if an o-graph $\Gamma$ represents an oriented manifold $M$ then the manifold $-M$ obtained by reversing the orientation of $M$ is represented by the o-graph $-\Gamma$ obtained from $\Gamma$ by switching overstrands and understrands at vertices, and changing each edge-colour to its opposite in $\mathbb{Z} / 3$. Now assume $M$ belongs to $\mathcal{M}_{n}$ and $\Gamma$ represents the unique minimal spine of $M$. Then $M$ is amphichiral if and only if $-\Gamma$ defines the same oriented polyhedron as $\Gamma$, which can be checked in $n^{2}$ time using an algorithm based 
on C-moves. Turning to the $M$ 's based on $G_{n}$ and using the C-moves again, one can now prove the following:

Proposition 2.10. If $\Gamma$ is the o-graph of Fig. 4 then $-\Gamma$ is a similar o-graph with colours:

$$
\begin{aligned}
& \alpha^{\prime}=1-\alpha, \quad \beta_{2 k+1}^{\prime}=1-\gamma_{2 k+1}, \quad \beta_{2 k}^{\prime}=1-\beta_{2 k}, \\
& \delta^{\prime}=1-\delta, \quad \gamma_{2 k+1}^{\prime}=1-\beta_{2 k+1}, \quad \gamma_{2 k}^{\prime}=1-\gamma_{2 k} .
\end{aligned}
$$

This proposition and Lemma 2.3 show that chirality for the elements of $\mathcal{M}_{n}$ obtained from the open chain $G_{n}$ can be tested in linear time: We only need to check whether one of four given $2 n$-tuples of elements of $\mathbb{Z} / 3$ coincides with another such given $2 n$-tuple.

\section{References}

[1] R. Benedetti and C. Petronio, A finite graphic calculus for 3-manifolds, Manuscripta Math., 88 (1995), 291-310, MR 96m:57008, Zbl 0856.57009.

[2] _ Combed 3-manifolds with concave boundary, framed links, and pseudoLegendrian links, J. Knot Theory Ramifications, 10 (2001), 1-35, MR 2002b:57010.

[3] B.G. Casler, An imbedding theorem for connected 3-manifolds with boundary, Proc. Amer. Math. Soc., 16 (1965), 559-566, MR 31 \#2730, Zbl 0129.15801.

[4] D.B.A. Epstein et al., Word Processing in Groups, Jones and Bartlett Publishers, Boston, 1992, MR 93i:20036, Zbl 0764.20017.

[5] A. Fomenko and S.V. Matveev, Algorithmic and Computer Methods for ThreeManifolds, Mathematics and its Applications, 425, Kluwer Academic Publishers, Dordrecht, 1997, MR 98k:57001, Zbl 0885.57009.

[6] R. Frigerio and C. Petronio, Construction and recognition of hyperbolic 3-manifolds with geodesic boundary, to appear in Trans. Amer. Math. Soc., Math.GT/0109012.

[7] M. Fujii, Hyperbolic 3-manifolds with totally geodesic boundary which are decomposed into hyperbolic truncated tetrahedra, Tokyo J. Math., 13 (1990), 353-373, MR 92a:57043, Zbl 0729.57005.

[8] D.J. Heath, On classification of Heegaard splittings, Osaka J. Math., 34 (1997), 497523, MR 99a:57014, Zbl 0885.57010.

[9] S. Kojima, Polyhedral decomposition of hyperbolic 3-manifolds with totally geodesic boundary, in 'Aspects of Low-Dimensional Manifolds, Kinokuniya, Tokyo', Adv. Stud. Pure Math., 20 (1992), 93-112, MR 94c:57023, Zbl 0802.57006.

[10] B. Martelli and C. Petronio, 3-manifolds up to complexity 9, Experiment. Math., 10 (2001), 207-236, MR 2002f:57045.

[11] S.V. Matveev, Complexity theory of three-dimensional manifolds, Acta Appl. Math., 19 (1990), 101-130, MR 92e:57029, Zbl 0724.57012.

[12] S.V. Matveev and T. Nowik, On 3-manifolds having the same Turaev-Viro invariants, Russian J. Math. Phys., 2 (1994), 317-324, MR 96a:57043, Zbl 0911.57014.

[13] Y. Miyamoto, Volumes of hyperbolic manifolds with geodesic boundary, Topology, 33 (1994), 613-629, MR 95h:57014, Zbl 0824.53038.

[14] V.G. Turaev and O. Viro, State sum invariants of 3-manifolds and quantum $6 j$ symbols, Topology, 31 (1992), 865-902, MR 94d:57044, Zbl 0779.57009. 
[15] A. Ushijima, A unified viewpoint about geometric objects in hyperbolic space and the generalized tilt formula, in 'Hyperbolic Spaces and Related Topics, II, Kyoto, 1999', Sūrikaisekikenkyūsho Kōkyūroku, 1163 (2000), 85-98, CMP 1799 371, Zbl 0969.51500.

[16] J.R. Weeks, Convex hulls and isometries of cusped hyperbolic 3-manifolds, Topology Appl., 52 (1993), 127-149, MR 95a:57021, Zbl 0808.57005.

Received July 3, 2002 and revised September 14, 2002.

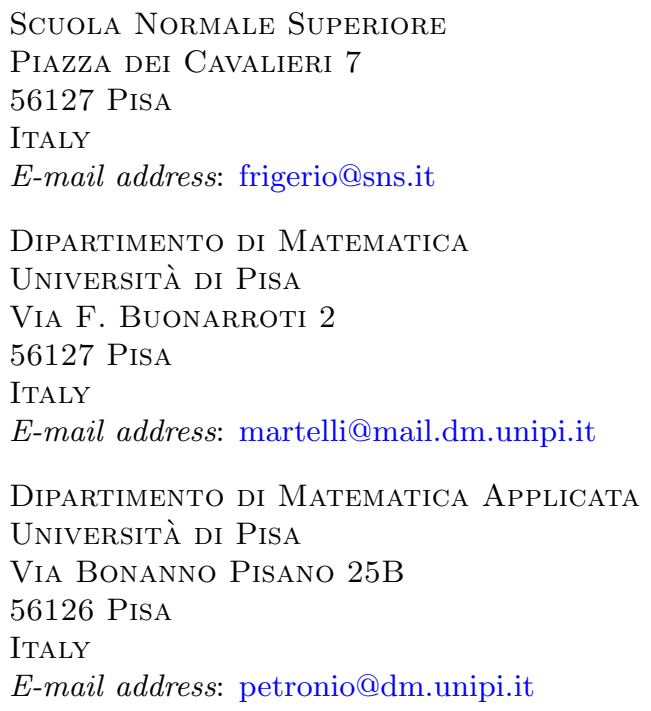

